\title{
Modeling international migration flows by integrating multiple data sources
}

\author{
Emanuele Del Fava ${ }^{1}$, Arkadiusz Wiśniowski ${ }^{2}$, and Emilio Zagheni ${ }^{1}$ \\ ${ }^{1}$ Max Planck Institute for Demographic Research, Rostock, Germany \\ ${ }^{2}$ School of Social Sciences, University of Manchester, Manchester, UK
}

\begin{abstract}
Migration has become a significant source of population change at the global level, with broad societal implications. Although understanding the drivers of migration is critical to enacting effective policies, theoretical advances in the study of migration processes have been limited by the lack of data on flows of migrants, or by the fragmented nature of these flows. In this paper, we build on existing Bayesian modeling strategies to develop a statistical framework for integrating different types of data on migration flows. We offer estimates, as well as associated measures of uncertainty, for immigration, emigration, and net migration flows among 31 European countries, by combining administrative and household survey data from 2002 to 2015 . Substantively, we document the historical impact of the EU enlargement and the free movement of workers in Europe on migration flows. Methodologically, our approach improves on the Integrated Modeling of European Migration (IMEM) framework by providing a robust statistical framework for evaluating recent migration trends that is flexible enough to be further extended to incorporate new data sources, like social media.
\end{abstract}

\section{Introduction}

To gain a better understanding of the causes and consequences of international population migration movements, migration scholars, official statisticians, and policymakers must overcome the inherent limitations of the various data sources that each country uses to produce statistics on migration, and especially on migration flows. These limitations include incompleteness and inconsistencies in the availability, definitions, and quality of the data (Willekens et al. 2016, Willekens 2019). A variety of sources are used to produce statistics on migration, including population censuses, population registers, household and passenger surveys, registers of foreigners, and 
special statistical forms - each of which has its characteristics and limitations (Martí \& Ródenas 2007, Kupiszewska \& Nowok 2008). Although all of these sources contain information related to migration, most are not explicitly designed to accurately measure migration. For these reasons, we 5 might expect to observe differences - and even quite large differences - in the numbers reported by these sources. In particular, we could expect to see discrepancies among data sources regarding the bilateral migration flows both within a country and between countries. Since these limitations might hamper the use of single sources to investigate migration, a possible solution to the problem of obtaining statistics on migration flows between pairs of countries is to combine the information from all of the available data sources. To accomplish this task, a statistical modeling framework can be used to build a "synthetic database" (Willekens 1994, 2019) that integrates migration data sources and auxiliary data in order to model migration flows 15 across time and estimate flows for which the available information is incomplete (Raymer et al. 2013). The Bayesian statistical approach can be used to express the level of trust in the available information (migration and auxiliary data) by means of probability distributions, to harmonize the data generated by different sources, and to provide measures of uncertainty for 20 both model parameters and predictions. Moreover, the Bayesian approach enables researchers to deal with the issue of data incompleteness by borrowing information from the available migration data and the auxiliary data to estimate the missing flows (Bijak \& Bryant 2016). The Integrated Modelling of European Migration (IMEM) project provided an excellent example of 25 such a synthetic database. It consisted of a Bayesian hierarchical model that was used to estimate bilateral migration flows between the European Union (EU) and the European Free Trade Agreement (EFTA) countries and the rest of the world (Raymer et al. 2013, Wiśniowski et al. 2016). This model included two modules. The first module was a "measurement error so model" that adjusted the migration flow data for effects in order to capture different types of inconsistencies in the data. The second module was a theory-driven "migration model" that used auxiliary information on the degree of attractiveness between countries (i.e., country- and dyad-specific demographic and socioeconomic covariates) to estimate the true migration flows amongst those countries.

In this paper, we propose extending the hierarchical Bayesian model developed within the IMEM project by combining official aggregated data on the number of international migration events in Europe, measured at both the origin and the destination, with individual-level data on transi40 tions to a new country drawn from national household surveys, such as the EU Labour Force Survey (LFS). The purpose of this data source integration is to obtain more realistic and accurate estimates, especially when the LFS data are able to capture migration flows that are not reflected in official statistics. Hence, our work innovates in three directions. First, we gen- 
eralize previous work on using LFS data to estimate migration flows from Poland to the UK (Wiśniowski 2017) by extending this approach to all of the EU/EFTA countries in which the survey is carried out. Second, the integration of multiple migration data sources requires the definition of source- and 5 country-specific measurement error models, in order to accommodate the characteristics of each data source. Third, we modify the measurement error models introduced by Raymer et al. (2013) for the migration event data, and by Wiśniowski (2017) for the LFS data, by linking them through a common underlying migration parameter called the "relocation rate" (Nowok \& Willekens 2011), which is derived through the migration model. We apply our model to a set of countries made up of the $28 \mathrm{EU}$ countries and three EFTA countries (we exclude Lichtenstein, for which neither the LFS data nor some auxiliary data are available) using migration flow data from 2002 to 2015. We then estimate the true latent migration flows among these countries, together with the associated measures of uncertainty. As more and more survey data with migration information will become available globally, our methods can be expanded to a number of different contexts.

The paper is organized as follows. In Section 2, we discuss the main sources of data on international migration flows that are available in the European context (population censuses, administrative data, household surveys), as well as their drawbacks, including their incompleteness and inconsistencies. In Section 3, we describe the Bayesian methodology used to model the data. In Section 4, we present the results obtained with the Bayesian model, describing in more detail the findings for three countries used as case studies. Finally, in Section 5, we wrap up the main points of the paper and present some ideas for future research.

\section{Data}

Migration data - i.e., data on either the stock of migrants or migration flows - can be obtained from different sources, each of which has its own characteristics and potential drawbacks.

\subsection{Census data}

A primary source of data on migration, especially for stocks, is the population census, which typically includes information on the country of birth and the country of citizenship of each respondent (United Nations Statistics 35 Division 1998).

While these data provide information on stocks, using them to estimate flows is not straightforward because the information on the migrants' previous country of residence is usually not collected. Moreover, even when this information is collected - e.g., when respondents are asked about the place 
of residence at a specified date in the past, usually one or five years preceding the census - its use for monitoring migration flows may be hindered by the fact that censuses are typically carried out every ten years and that people may relocate more than once in the period between two censuses.

A solution that has been developed to tackle the lack of explicit information in census data on migration flows uses demographic accounting methods to estimate the flows that are consistent with the differences in migrant stocks between two consecutive censuses, while also taking into account the total numbers of births, deaths, and net migrations that occur between two censuses (Abel 2013, Abel \& Sander 2014, Azose \& Raftery 2019, Abel \& Cohen 2019).

For the analyses presented in this paper, we will not use data from population censuses. It is, however, critical that we envisage ways to include such information in studies of migration flows, especially when other sources of data are lacking.

\subsection{Administrative data}

Administrative data, which are derived from population registers, registers of foreigners or resident permits, sample surveys, specific statistical forms or other administrative sources, represent a better source of information than 2 census data, as they can capture residence changes when they occur or are declared. However, for several reasons, comparing the administrative data of different countries can be difficult (Abel 2010, Raymer et al. 2013, Willekens 2019).

One potential problem that can arise when comparing these data is bias due to the under-registration of migrants. This issue is particularly salient for emigration and return migration flows, given that immigrants generally have incentives or obligations to register as a resident in the country of destination, but people moving abroad usually have few incentives to deregister (and, subsequently, to register again if they are returning). The problem of undercounting is exacerbated in surveys (such as the International Passenger Survey in the UK), as surveys often lack statistical precision due to their small sample sizes, and because migration transitions are rare events that are hard to capture in such surveys (Martí \& Ródenas 2007).

A second issue that may come up in international comparisons of administrative data is related to the differences in the duration criteria used by different countries to identify international migrants. According to the UN definition (United Nations Statistics Division 1998), long-term international migrants are those who relocate from their country of usual residence to a different country for a minimum stay of 12 months, while short-term migrants are those who live abroad for three to 12 months. However, countries may use different duration thresholds for identifying migrants, and often use the intended stay duration as an approximation of the actual length of 
stay. For instance, Germany and Spain have no time criteria at all, which means that all individuals who enter either of these countries for purposes other than tourism or business are required to register. In other countries, an immigrant is defined as a person who registers for a stay of at least three 5 months (e.g., Austria and Slovenia), six months (e.g., Denmark and Norway), one year (e.g., Italy and Netherlands), or permanently (e.g., Czechia and Poland).

Following the EU Regulation (EC) No 826/2007, European countries have started to transition to adopting the UN definition. Under the regulation, the current definition of migration requires countries to record as immigrants those individuals who stay in the country for at least one year. Therefore, each country has to submit data for reference year $t$ to Eurostat at the end of year $t+1$, with dissemination occurring in February $t+2$. For instance, data referring to the year 2017 had to be submitted to Eurostat at the end of 2018, and were successively made publicly available from February 2019 onward. Moreover, since 2008, countries have been using additional sources or statistical modeling, striving to improve the quality of the statistics transmitted to Eurostat, such as health insurance registers, tax registers, 2011 census data, and estimation methods based on the GDP or

20 on a residency index (e.g., Estonia (Maasing et al. 2017)). In addition, some countries are using the mirror flows reported by partner countries to correct coverage errors (personal communication with a Eurostat representative).

The third potential problem with using register data is related to the accuracy of data collection systems. The five Nordic countries (Denmark, Norway, Sweden, Finland, and Iceland) have high-quality, harmonized population registers that routinely exchange information. However, a number of countries use less reliable sources, such as registers of foreigners (e.g., Hungary and Slovakia), statistical forms (e.g., Poland and Malta), or surveys (e.g., Portugal and UK). Finally, some countries do not use population registers at all to measure international mobility, as they do not record the country of origin of individuals who have changed residence (e.g., France and Greece).

Despite these issues, the IMEM project has shown how Bayesian hierarchical modeling can be used to harmonize administrative data on migration and to estimate the true latent flows, even for those countries with no available data (Raymer et al. 2013, Wiśniowski et al. 2016)).

\subsection{Household survey data}

Another data source that can be used to study migration within Europe is the European Union Labour Force Survey (EU LFS or, simply, LFS), which is a large household survey that provides quarterly and yearly results on the labor market participation of people aged 15 or older, and on people outside the labor force. The LFS is carried out in all $28 \mathrm{EU}$ countries and 
in three EFTA countries (Lichtenstein is excluded), as well as in four candidates countries (Montenegro, North Macedonia, Serbia, and Turkey). Even though these surveys are designed to measure labor migration, they may capture more general forms of migration, as information on immigration is

5 collected for all members of the selected households. The LFS questionnaire asks each participant questions, and the responses to these questions can, in principle, be used to produce statistics on migrant stocks (nationality and country of birth of the participant) and on migration flows (country of residence of the participant one year before the survey). However, problems can arise when the LFS data are used to estimate migration without applying any data correction (Martí \& Ródenas 2007).

It is, for example, important to note that these data generally lack statistical precision. Since migrants represent a tiny part of the total population of a country, the survey sample may not be large enough to capture them.

Moreover, there may be issues of statistical bias related to how frequently the survey sample is updated. Three types of problems are particularly likely to arise. First, the less frequently the sampling list is updated with new entries, the less able the survey will be to capture new migrations, as the participants who remain in the sample for several waves do not provide new information on migration. Second, if the survey does not include collective households, the size of certain migrant subgroups (e.g., military personnel, students, or members of religious communities) may be underestimated. Third, in those countries in which survey participation is not mandatory, rates of non-response may be relatively high for a number of reasons, including a lack of interest in the survey, language barriers, or the fear of providing personal information, especially among undocumented migrants.

Thus, unless specific adjustments are made to account for these issues, LFS data might underestimate migration stocks, and, to an even greater extent, migration flows.

\section{Methods}

In this section, we introduce the hierarchical Bayesian statistical framework for modeling the migration flows among pairs of European countries, integrating data from multiple data sources (administrative data on immigration and emigration and the LFS). We use a migration model to estimate the true latent bilateral migration flows from country $i$ to country $j$ in year $t, Y_{i j t}^{12}$, conditional on the definition of long-term migration as a relocation followed by a minimum duration of stay of 12 months. In order to account for differences in data measurement among countries and data sources, our statistical framework includes a measurement error model. The issue of data incompleteness is mainly tackled by using a migration model to estimate the missing data. Our statistical model extends the methodology separately de- 
veloped by Nowok \& Willekens (2011), Raymer et al. (2013), and Wiśniowski (2017).

In the next subsections, we present in more detail both the measurement error model and the migration model.

\section{3.1 Measurement error model}

The purpose of the measurement error model is to estimate the true latent number of relocations (change of usual residence, with no information on the duration of stay), while accounting for the inconsistencies among the data sources related to undercounting, population coverage, and the accuracy of the data collection system. Thus, the model specification differs among the data sources to account for their specific characteristics and limitations. In Sections 3.1.1 and 3.1.2, we present the models for the administrative data and the LFS data, respectively.

\subsubsection{Administrative data}

Data on international migration flows from administrative data sources which are mainly obtained from population registers at the local or the central level, but also from other sources, such as registers of foreigners or sample surveys; and which are disseminated by Eurostat - consist of counts of migration events $x_{i j t}^{k}$ from country $i$ to country $j$ in year $t$, provided by data source $k$, which is either on immigration by country of previous residence (IR), or on emigration by country of next residence (ER).

A typical distribution for count data is the Poisson distribution, which implies that the mean is equal to the variance. However, we believe that this assumption does not hold for these data, as they exhibit high levels of uncertainty both within each country and between countries because of problems with undercounting, population coverage, and the accuracy of the data collection system. Hence, to account for the high degree of uncertainty associated with migration data, we assume that the number of migration events is distributed according to a Poisson-Log normal distribution; i.e., 30 $x_{i j t}^{k}$ is distributed according to a Poisson distribution with parameter $\lambda_{i j t}^{k}$, the expected number of migration events,

$$
x_{i j t}^{k} \sim \operatorname{Po}\left(\lambda_{i j t}^{k}\right)
$$

and $\lambda_{i j t}^{k}$, in turn, follows a log-normal distribution with mean $\zeta_{i j t}^{k}$ and dispersion $\tau_{i j t}^{k}$ :

$$
\lambda_{i j t}^{k} \sim L N\left(\zeta_{i j t}^{k}, \tau_{i j t}^{k}\right) .
$$

For the mean $\zeta_{i j t}^{k}$, we specify the following model for both immigration and 35 emigration data:

$$
\zeta_{i j t}^{I R}=\log Y_{i j t}+\log v_{f^{I R}(j)}-\log \left(1+e^{-\kappa_{j}}\right),
$$




$$
\zeta_{i j t}^{E R}=\log Y_{i j t}+\log v_{f E R(i)}-\log \left(1+e^{-\kappa_{i}}\right) .
$$

The two equations in Eq. 3 express the idea that the data originate from measuring the true latent number of migration events, $Y_{i j t}$, that these measurements are biased due to issues of undercounting and incomplete coverage, and that these sources of bias can be accommodated with specific effects 5 for the level of undercounting (depending on $v_{f^{I R}(j)}$ with the immigration data, or on $v_{f E R(i)}$ with the emigration data), and for the population coverage (depending on $\kappa_{j}$ with the immigration data, $\kappa_{i}$ with the emigration data), respectively (more details on the parameters are given in the next subsections). Moreover, the precision $\tau_{i j t}^{k}$ in Eq. 2 quantifies the uncertainty in the data; is independent of the mean $\zeta_{i j t}^{k}$ (unlike in the Poisson distribution); and is a measure of the accuracy of the data source. Overall, the inclusion of the effects for undercounting, population coverage, and data source accuracy enables us to take into account the overdispersion in the data.

Log-linear models are a standard choice for the analysis of international migration flows, as these models have previously been used to parametrize the classical gravity model for mobility as a Poisson regression model under the generalized linear modeling (GLM) framework (Cohen et al. 2008). Thus, the modeling framework introduced in Eq. 3 for the mean $\zeta_{i j t}^{k}$ can be rewritten as a log-linear model for the parameter $\lambda_{i j t}^{k}$ :

$$
\begin{aligned}
\log \lambda_{i j t}^{I R} & =\log Y_{i j t}+\log v_{f^{I R}(j)}-\log \left(1+e^{-\kappa_{j}}\right)+\frac{\varepsilon_{i j t}^{I R}}{\tau_{f^{I R}(j)}} \\
\log \lambda_{i j t}^{E R} & =\log Y_{i j t}+\log v_{f E R(i)}-\log \left(1+e^{-\kappa_{i}}\right)+\frac{\varepsilon_{i j t}^{E R}}{\tau_{f E R}(i)} .
\end{aligned}
$$

In Eq. 4, we rewrote $\tau_{i j t}^{k}$ as $\tau_{f^{k}(j)}$ to express the dependency on the functions $f^{I R}(j)$ and $f^{E R}(i)$; we also introduced the parameters $\varepsilon_{i j t}^{I R}$ and $\varepsilon_{i j t}^{E R}$, which are independent error terms that follow a standard normal distribution, $N(0,1)$. In the following subsections, we provide an explanation for each term on the right-hand side of Eq. 4.

True number of migrations. The true latent number of migrations $Y_{i j t}$ is equal to the number of true latent relocations from $i$ to $j, R_{i j t}^{R}$, conditional on a minimum continuous stay in country $j$ for a period of $d_{m}$ years (Nowok \& Willekens 2011). In a context with more than two countries, we can derive $Y_{i j t}$ using the following equation:

$$
Y_{i j t}=R_{i j t}^{R} \exp \left(-\mu_{j+t} d_{m}^{j}\right)=\mu_{i j t} N_{i t} \exp \left(-\mu_{j+t} d_{m}^{j}\right),
$$

where $\mu_{i j t}$ denotes the relocation rate from country $i$ to country $j$ in year $t$, $N_{i t}$ denotes the population of the country of origin exposed to the possibility of migrating (we assume that every person in the country of origin is at risk 
of relocating to a different country), and the factor $\exp \left(-\mu_{j+t} d_{m}^{j}\right)$, where $\mu_{j+t}=\sum_{i ; i \neq j} \mu_{j i t}$, accounts for the survival in country $j$ for a minimum duration of stay equal to $d_{m}^{j}$ (Nowok 2010). For modeling purposes, we can rewrite Eq. 4 to make explicit the dependence of the true latent number of 5 migrations on the relocation rate:

$$
\begin{aligned}
\log \lambda_{i j t}^{I R} & =\log R_{i j t}^{R}-\sum_{i ; i \neq j} \mu_{j i t} d_{m}^{j}+\log v_{f^{I R}(j)}-\log \left(1+e^{-\kappa_{j}}\right)+\frac{\varepsilon_{i j t}^{I R}}{\tau_{f^{I R}(j)}} \\
\log \lambda_{i j t}^{E R} & =\log R_{i j t}^{R}-\sum_{i ; i \neq j} \mu_{j i t} d_{m}^{j}+\log v_{f^{E R}(i)}-\log \left(1+e^{-\kappa_{i}}\right)+\frac{\varepsilon_{i j t}^{E R}}{\tau_{f^{E R}(i)}}(6)
\end{aligned}
$$

To facilitate the interpretation of Eq. 6, we can take the exponential function on both sides of the equation to obtain the following multiplicative model on the linear scale:

$$
\begin{aligned}
\lambda_{i j t}^{I R} & =R_{i j t}^{R} \exp \left(-\sum_{i ; i \neq j} \mu_{j i t} d_{m}^{j}\right) \frac{v_{f^{I R}(j)}}{1+e^{-\kappa_{j}}} \exp \left(\frac{\varepsilon_{i j t}^{I R}}{\tau_{f^{I R}(j)}}\right), \\
\lambda_{i j t}^{E R} & =R_{i j t}^{R} \exp \left(-\sum_{i ; i \neq j} \mu_{j i t} d_{m}^{j}\right) \frac{v_{f^{E R}(i)}}{1+e^{-\kappa_{i}}} \exp \left(\frac{\varepsilon_{i j t}^{E R}}{\tau_{f E R(i)}}\right) .
\end{aligned}
$$

The factor $d_{m}^{j}$ in Eq. 6 (and Eq. 7) is expressed in years, and can be equal to zero (as there is no time limit, each relocation is considered a migration), 0.25 years (three months), 0.5 years (six months), one year (12 months; i.e., the reference period chosen by the United Nations and the EU Regulation No. 862/2007 in their recommendation for defining long-term migration), or five years or more (permanent residence) (Nowok 2007).

Undercounting. The second term in Eq. 7, $v_{f^{k}(j)}$, accounts for the systematic bias due to the undercounting of the migration events in the data source $k$ (Raymer et al. 2013). The parameter $v$ ranges between zero and one on the linear scale, with values closer to one indicating low levels of undercounting and values closer to zero indicating high levels of undercounting.

Depending on the indicators $f^{k}(j)$, we differentiate the parameter between countries assumed to have low levels of undercounting $(f=0)$ or high levels of undercounting $(f=1)$. To simplify the notation, we denote the parameters $v_{f^{I R}(j)=0}$ and $v_{f^{I R}(j)=1}$ as $v_{1}$ and $v_{2}$, respectively; similarly, we denote the parameters $v_{f E R(i)=0}$ and $v_{f E R(i)=1}$ as $v_{3}$ and $v_{4}$, respectively. The classification of countries by whether they have low or high levels of undercounting is based on information elicited from expert opinion (Raymer \& Wiilekens 2008, Raymer et al. 2013). For instance, we expect that rates of undercounting will be higher in countries where the administrative data depend on self-declaration. Moreover, we expect $v_{f^{k}(j)}$ to be closer to zero 
for emigration than for immigration, since there are usually more personal and social benefits associated with registering than with deregistering.

To estimate the parameters $v_{f^{k}(j)}$, we specified a prior distribution, which is used in the Bayesian framework to express our belief or previous 5 information in probabilistic terms. This prior distribution, combined with the Poisson-Log normal likelihood of data, enables us to derive the posterior distribution of each parameter of interest (as well as the missing data). The posterior distribution of each parameter can be summarized by taking the median and constructing credible intervals (CIs), such as a 50\% CI (ranging between the $25 \%$ and the $75 \%$ quantiles) and a $95 \%$ CI (ranging between the $2.5 \%$ and the $97.5 \%$ quantiles).

We specify a Beta prior for $v_{f^{k}(j)}$, which is a continuous distribution (with shape parameters $\alpha$ and $\beta$ ) ranging from zero to one, and is, therefore, exactly like the parameter $v$. Since the prior distributions that were based on the elicited expert opinion (Wiśniowski et al. 2013) and were used in the IMEM model proved to be rather weakly informative because of the high degree of uncertainty in expert opinion (Willekens 2019), we decided to specify our own priors based on our beliefs, which are also associated with a high level of uncertainty. To express our belief that the degree of undercounting is lowest for the immigration to the countries classified as having low levels of undercounting, we define for the parameter $v_{1}$ a Beta prior centered around 0.8 with standard deviation equal to 0.1 : namely, $\operatorname{Beta}(12,3)$, which implies that the parameter ranges a priori within a $95 \%$ CI between 0.57 and 0.95. At the other extreme, since we believe that 25 the degree of undercounting is highest for emigration from the countries classified as having high levels of undercounting, we define for the parameter $v_{4}$ a Beta prior centered around 0.4 with standard deviation equal to 0.1: namely, Beta $(9.2,13.8)$, which implies a parameter ranging a priori within a $95 \%$ CI between 0.21 and 0.60. Finally, to express our uncertainty for the cases with high levels of undercounting for immigration data $\left(v_{2}\right)$ and low levels of undercounting for emigration data $\left(v_{3}\right)$, we define for both groups a Beta prior centered around 0.6 with standard deviation equal to 0.1: namely, Beta $(13.8,9.2)$, which implies a parameter ranging within a $95 \%$ interval between 0.40 and 0.79 .

35 Coverage. The third term in Eq. $7,1 /\left(1+e^{-\kappa_{j}}\right)$ (which can also be written as $\left.\operatorname{logit}^{-1}(\kappa)\right)$, adjusts for the population coverage of the data collection system in the country of destination (model IR) or in the country of origin (model ER) (Raymer et al. 2013). Since this term is assumed to range between zero (very poor coverage) to one (optimal coverage) on the linear 40 scale, it is expressed as a logistic function depending on the parameter $\kappa$, which is a country-specific and normally distributed random effect. Hence, higher positive values of $\kappa$, so that $1 /\left(1+e^{-\kappa_{j}}\right)$ tends to one, imply good coverage, while higher negative values, so that $1 /\left(1+e^{-\kappa_{j}}\right)$ tends to zero, imply poor coverage. 
We distinguish between countries with "standard" coverage, for which $\kappa$ is distributed according to the normal distribution $N(\nu, \eta)$, and countries with "excellent" coverage (the Nordic countries plus the Netherlands), for which the term $1 /\left(1+e^{-\kappa_{j}}\right)$ is assumed to be equal to one, which implies 5 perfect coverage according to Raymer et al. (2013). For the distribution of the hyperparameters $\nu$ and $\eta$, we follow the specification chosen by Raymer et al. (2013), setting $\nu \sim N(0,0.05)$ and $\eta \sim \Gamma(4,1)$. This implies that, for countries with standard coverage, we give $\kappa$ a weakly-informative prior distribution, since it implies that $\kappa$ ranges a priori between -9 and 9 ; which, in turn, means that $1 /\left(1+e^{-\kappa_{j}}\right)$ ranges between zero and one on the linear scale.

Accuracy. Finally, the term $\varepsilon / \tau_{f^{k}}$ is an error term, where the parameter $\varepsilon$ follows a standard normal distribution, $N(0,1)$, and the precision parameter $\tau_{f^{k}}$ accounts for the accuracy of the data collection system (Raymer et al. 2013). If the precision $\tau_{f^{k}}$ is high, the whole error term will be smaller, indicating a higher level of accuracy. On the other hand, lower values of $\tau_{f^{k}}$ will inflate the error term, which is evidence of a lower level of accuracy.

We use prior knowledge to group the countries based on the accuracy of the data collection system for immigration or emigration, as the precision $\tau_{f^{k}}$ are considered to be group-specific (Raymer et al. 2013). In absence of further information, we assume that this grouping is time-invariant, even though it may expected that changes in the collection systems over time might imply a change in the grouping composition. The first group, which is characterized by the precision $\tau_{f^{k}(j)=1}$, includes the Nordic countries, which are assumed to have very high levels of accuracy, as they exchange information on migration flows among themselves. The second group, which is characterized by the precision $\tau_{f^{k}(j)=2}$, includes countries with reliable data collection systems. Finally, the third group, which is characterized by the precision $\tau_{f^{k}(j)=3}$, is made up of the remaining countries, which have less reliable systems for collecting migration data.

For all these $\tau_{f^{k}(j)}$ parameters, we specify weakly-informative priors: namely, $\Gamma(0.01,0.01)$, which assume that the precision parameters are centered around one and are spread over a very large positive range. Like in the case of the undercounting parameters, the choice of weakly-informative priors is motivated by the finding that the elicited expert opinion in the IMEM project contributed very little to the prior information (Wiśniowski et al. 2013).

\subsubsection{Survey data}

Unlike the administrative data sources, the EU LFS provides data on transitions over a one-year period. The LFS can provide information not only on migrant stocks by nationality and country of birth, but on immigration flows, as the survey asks participants to name the country where they were 
living one year before the survey was conducted (Martí \& Ródenas 2007). We believe that one year is short enough to allow us to assume that the individuals who relocated made at most one transition from the country of origin to the country of destination. This "naive assumption" (Schmertmann 5 1999) implies that the observed transitions correspond to all of the relocations undertaken by the survey participants in the given year. This, in turn, implies that the number of transitions coincides with the number of events, and is, therefore, consistent with the information provided by the administrative data sources. In line with the assumptions we made about the counts of migration events from the administrative sources in Section 3.1.1, we assume that the counts of transitions obtained from the LFS in the country of destination (IS), $k_{i j t}$, follow a Poisson distribution with parameter $\lambda_{i j t}^{I S}$, which is the expected number of transitions from country $i$ to country $j$ that occurred between year $t-1$ and year $t$. This parameter follows, in turn, a log-normal distribution with the mean $\xi_{i j t}$ and the precision $\omega_{i j t}$ :

$$
\log \lambda_{i j t}^{I S} \sim L N\left(\xi_{i j t}, \omega_{i j t}\right) .
$$

For the mean $\xi_{i j t}$, we specify the following model depending on the true latent number of transitions $R_{i j t}^{S}$ and on additional effects for the bias in measurement:

$$
\xi_{i j t}=\log R_{i j t}^{S}+\log \frac{n_{j t}}{N_{j t}}+\log v_{g^{I S}(j)} .
$$

Eq. 9 can also be rewritten as a log-linear model for the parameter $\lambda_{i j t}^{I S}$, 20 namely,

$$
\log \lambda_{i j t}^{I S}=\log R_{i j t}^{S}+\log \frac{n_{j t}}{N_{j t}}+\log v_{g^{I S}(j)}+\frac{\varepsilon_{i j t}^{I S}}{\omega_{g^{I S}(j)}} .
$$

The ratio between the sample size $n_{j t}$ and the population $N_{j t}$ in country $j$ is the inclusion probability of the survey - corresponding to the householdspecific "yearly coefficient" - and represents the probability of a household being included in the sample. In the limit case in which $n_{j t}=N_{j t}$ and the survey is actually a census, the inclusion probability is equal to one and the expected number of transitions $\lambda_{i j t}^{I S}$ is equal to the true number of transitions $R_{i j t}^{S}$ (if we do not consider the effects of the undercounting and the accuracy of the data collection system). However, as in most cases $n_{j t} \ll$ $N_{j t}$, the inclusion probability is smaller than one and the term $\log \left(n_{j t} / N j t\right)$ is negative, which implies that the measured data are a subset of the whole number of transitions. To facilitate the interpretation of Eq. 10, we can again take the exponential function of both sides of the equation to obtain the following multiplicative model on the linear scale:

$$
\lambda_{i j t}^{I S}=R_{i j t}^{S} \frac{n_{j t}}{N_{j t}} v_{g^{I S}(j)} \exp \left(\frac{\varepsilon_{i j t}^{I S}}{\omega_{g^{I S}(j)}}\right) .
$$


Undercounting. The third term in Eq. 11, $v_{g^{I S}(j)}$, accounts for the systematic bias due to the undercounting of events as a consequence of the survey design.

We classify as low-undercounting $\left(g^{I S}(j)=0\right)$ those countries where par-

5 ticipation in the LFS survey is mandatory by law (10 out of 31 EU/EFTA countries), while we classify as high-undercounting $\left(g^{I S}(j)=1\right)$ those countries where participation is voluntary. In the latter group of countries, a migrant selected to participate in the survey might refuse due to language barriers, a lack of interest in the survey, or fear of being asked sensitive questions. For these reasons, the chances of capturing migration flows are lower when survey participation is voluntary than when it is mandatory. As in the model for the administrative data (Eq. 7), the parameter $v_{g^{I S}(j)}$ ranges between zero and one, with values closer to one indicating a low level of undercounting, and values closer to zero indicating a high level of undercounting. To simplify the notation, we denote the parameters $v_{g^{I S}(j)=0}$ and $v_{g^{I S}(j)=1}$ as $v_{5}$ and $v_{6}$, respectively.

To identify these parameters, we assume that the low undercounting parameter is larger than the high undercounting parameter. For the parameter $v_{5}$, we specify the prior $\operatorname{Beta}(13.3,4.4)$, which is centered around a mean of 0.75 (with standard deviation 0.1 ), and varies a priori within the $95 \%$ CI between 0.53 and 0.92 . Instead, for the parameter $v_{6}$, we use the prior $\operatorname{Beta}(4.4,13.3)$, which is centered around a mean of 0.25 (with standard deviation 0.1), and varies a priori within the $95 \%$ CI between 0.09 and 0.4 .

Accuracy. The nuisance term $\varepsilon / \omega_{g^{k}}$ in Eq. 11 consists of an error term $\varepsilon$, which follows a standard normal distribution, and a precision parameter $\omega_{g^{k}}$. As in the model for the administrative data, we find that the larger the precision $\omega_{g^{k}}$ is, the smaller the whole error terms are and the higher the level of accuracy is.

We assign each country to one of two groups depending on how accurate the survey is in capturing the country's new migrants year after year, which we assume depends on how frequently the country updates the sample composition. According to Martí \& Ródenas (2007), each country is characterized by a specific rate of impossible answer, which refers to a situation that occurs when participants remain in the survey for more than one year, and thus prevent more recent migrants from entering the sample. The first group is made up of countries with a rate of impossible answer lower than $50 \%$, and a level of precision denoted by $\omega_{g^{I S}(j)=1}$. The countries in this group change their sample composition more frequently, and may therefore 40 be more accurate in capturing new transitions.

The second group is made up of countries with an impossible answer rate ranging between $51 \%$ and $75 \%$, and a level of precision denoted by $\omega_{g^{I S}(j)=2}$. Since these countries change their sample composition less frequently and the participants remain in the panel for more than one year, we expect that 
their level of accuracy in capturing new migrants will be lower than that of the first group.

For both $\omega_{g^{I S}(j)=1}$ and $\omega_{g^{I S}(j)=2}$ parameters, we specify priors distributions that are weakly-informative: namely, $\Gamma(0.01,0.01)$, which assume the

5 parameters to have a mean of one and a very large variance.

\subsection{Migration model}

The second component of the hierarchical Bayesian model consists of a migration model, which we use to derive the true latent migration flows. Although we specify our model as a gravity model of migration, our aim is the assess the effect of a set of chosen covariates on the latent migration flows, as would be the case if a regression model were applied. Rather, we use the stochastic process induced by the Bayesian approach to predict the missing data, and, in turn, to derive the true latent migration flows. The variables included in the model have been chosen based on both migration theory and empirical evidence, and have been shown to provide relevant information on the socioeconomic and demographic factors that influence the relative attractiveness of pairs of countries (Abel 2010, Raymer et al. 2013).

In contrast to the approach we used to formulate the measurement error models, in which we defined a model for each data source to account for the specific characteristics of the data, we have chosen to develop a single migration model for all of the data sources, given that the true migration flow underlying the multiple data sources is the same.

We assume that the true latent number of relocations from the administrative data sources, $R_{i j t}^{R}$, and the true latent number of transitions from the survey data, $R_{i j t}^{S}$, originate from two log-normal distributions with a common mean $\mu_{i j t}^{Y}$ (because the underlying migration flows are the same) and source-specific precisions, $\tau^{R}$ and $\tau^{S}$ (because the data sources do not capture the migration flows in the same way). We then define the following migration model for the common mean $\mu_{i j t}^{Y}$, with the period under study being from 2002 to 2015 :

$$
\begin{aligned}
\mu_{i j t}^{Y}= & \gamma_{1}+\gamma_{2} \log N_{i t}+\gamma_{3} \log N_{j t}+\gamma_{4} \log D_{i j}+\gamma_{5} \log \frac{G_{j t}}{G_{i t}}+ \\
& \gamma_{6} A_{i j t}+\gamma_{7} A_{i t}+\gamma_{8} A_{j t}+\gamma_{9} \log I_{i j t}+\gamma_{10} \log E_{i j t}+\gamma_{11} \log M_{i j}+ \\
& \gamma_{12} \log M_{j i}+\gamma_{13} \log L_{i j}+\gamma_{14} F_{i j t}+\sum_{k=15}^{27} \gamma_{k} P_{t}+u_{i j} .
\end{aligned}
$$

The migration model in Eq. 12 contains the following variables:

1. The population of both the country of origin, $N_{i t}$, and the country of destination, $N_{j t}$. (Source: Eurostat.) 
2. The international (and intra-national) bilateral distance between the country of origin and the country of destination, denoted as $D_{i j}$, which depends on the bilateral distance between the biggest cities of the two countries (Mayer \& Zignago 2011).

3. The ratio of the Gross National Income (GNI) per capita in the destination country, denoted as $G_{j t}$, to the GNI of the country of origin, denoted as $G_{i t}$. (Source: World Development Indicators, World Bank).

4. Three indicators variables for EU/EFTA membership status between 2002 and 2017. The first variable, $A_{i j t}$, takes a value of one if both countries $i$ and $j$ were members of the EU/EFTA in year $t$. The second indicator, $A_{i t}$, takes a value of one if the origin country $i$ was a member of the EU/EFTA in year $t$. The third indicator, $A_{j t}$, takes a value of one if the destination country $j$ was a member of the EU/EFTA in year $t$.

5. The international trade between the country of origin $i$ and destination $j$ in year $t$, expressed as imports in EUR, $I_{i j t}$ and exports in EUR, $E_{i j t}$. (Source: Eurostat, EU/EFTA trade by SITC).

6. The bilateral migrant stocks by country of birth around the year 2000, based on population censuses. The variable $M_{i j}$ stands for the migrant stock born in the country of origin $i$ and living in the country of destination $j$, and is used to capture the pull effect of the migrant network residing in the destination. The variable $M_{j i}$ stands for the migrant stock born in the country of destination $j$ and living in the country of origin $i$, and is used to capture the push effect from the returning migrants in the country of origin (Özden et al. 2011).

7. An index of Common Language (CL), denoted as $L_{i j}$, ranging between zero and one and obtained by aggregating three indices on Common Official Language (COL), Common Native Language (CNL), and Language Proximity (LP). An index closer to one indicates a higher degree of commonality between the languages of the origin and the destination countries (Melitz \& Toubal 2014).

8. An indicator variable, $F_{i j t}$, taking a value equal to one for the year $t$ in which there is freedom of movements for workers from country $i$ in country $j$; meaning that these migrants can take up any employment under the same conditions as those that apply to the nationals (Source: European Commission).

9. Indicator variables for the time effect for the years 2002-2014, denoted as $P_{t}$ with $t=1, \ldots, 13$, to capture the time pattern of migration flows over the years. The reference year is 2015 . 
10. A random effect $u_{i j}$ to smooth the data over time by capturing the dyadic effect of each pair of bilateral migration flows between two countries. These random effects are normally distributed with a mean of $v_{i j}$ and precision $\tau_{u}, u_{i j} \sim N\left(v_{i j}, \tau_{u}\right)$. The hyperparameters $v_{i j}$ are data source should be weighted more by specifying for the parameter $w_{R}$ the prior Beta $(12,12)$, which is centered around a mean of 0.5 (with standard deviation 0.1 ) and varies a priori within the $95 \%$ CI interval between 0.30 and 0.70 .

True number of migration flows. Finally, the true latent number of migration flows $Y_{i j t}^{12}$, conditional on a duration of stay of at least 12 months (in accordance to the recommendation of the United Nations and the EU Regulation (EC) No 862/2007), is generated using the estimate of the true latent relocation rate $\mu_{i j t}$ (Nowok 2010, Nowok \& Willekens 2011):

$$
Y_{i j t}^{12}=\mu_{i j t} \times N_{i t} \times \exp \left(-\mu_{j+t} \times d_{m}^{j}\right),
$$

where $d_{m}^{j}=1$ and the term $\mu_{j+t}=\sum_{i ; i \neq j} \mu_{j i t}$.

\section{Results}

We developed our Bayesian model in JAGS (Plummer 2003) using R software. We computed the summary statistics from the posterior distribution of the parameters with MCMC samples of 8,000 iterations, using four chains 
of 30,000 iterations each (10,000 iterations of burn-in and a thinning of 10 ; i.e., we saved each $10 t h$ iteration). The convergence of the monitored parameters was assessed using standard diagnostics present in the package coda (Plummer et al. 2006).

\section{$5 \quad 4.1 \quad$ Model results}

We ran the model on a set of 31 EU/EFTA countries, excluding Lichtenstein (LI) because of the lack of LFS data for this country. We summarize the results of the measurement error model in Table 1, where we show the quantiles from the posterior distribution of each parameter.

Looking at the coverage parameters, $\operatorname{logit}^{-1}(\kappa)$ (equivalent to $1 /(1+$ $\left.e^{-\kappa_{j}}\right)$ ), we notice that for the countries with no data from the registers (Cyprus, France, Greece, Hungary, Latvia, Portugal, and Romania), the model was not able to identify the value of the parameter, as the posterior median, which is approximately equal to 0.80 , and the $95 \%$ CI ranging be15 tween 0.01 and one show. The consequence is an increase in the uncertainty around the estimates of the true latent number of migration events for these countries.

When we turn to the undercounting parameters for the administrative data, we see that the posterior characteristics of the parameters of the highundercounting groups, namely, $\lambda_{2}$ and $\lambda_{4}$, strictly reflect our assumption regarding the prior distribution, while the posterior characteristics for the low-undercounting parameters reflect the influence of the higher quality of the data. However, fine-tuning of the priors is still deemed necessary for the parameters for the survey data, given that, the two parameters are quite 25 different from those assumed based on the priors, and that it appears that there is no difference between the two groups - which, we recall, are based on either the voluntary or the mandatory participation in the survey.

The accuracy parameters showed that the highest levels of accuracy were in the Nordic countries, both for immigration, and, to a lesser extent, for emigration. By contrast, we estimated particularly low values for the level of accuracy in the countries with data collection systems classified as least reliable. The lowest values for the degree of precision were reported for the survey data. In this case as well, it appears that the information on the frequency of sample updates, which was used to classify the countries as having high or low levels of accuracy, was not associated with the degree of accuracy, as there was no difference between the parameters $\omega_{1}^{I S}$ and $\omega_{2}^{I S}$.

The results for the migration models are reported in Table 2, where we show the quantiles from the posterior distribution of each coefficient and the precision parameters of the $u$ random effects. Several variables showed "credible evidence for a non-zero effect" (Matthews 2019); meaning that the $95 \%$ CI of their coefficients did not contain the value of zero, or that, if it did, this was an extreme value (for instance, the population terms $N_{i t}$ and 
$N_{j t}$ ). A positive, credibly non-zero effect was found for the ratio of the GNI between the destination country and the origin country, which implies that the richer country was more attractive as a destination country. Moreover, a migration flow from $i$ to $j$ was found to be more likely when the origin

5 country was a member of the EU/EFTA $\left(A_{i t}\right)$, and especially when there was freedom of movement for the workers in both countries $\left(F_{i j t}\right)$. This can be seen an indication of the positive effects of both the EU enlargement and the expansion of economic freedom on the size of the intra-EU migration flows. We also found a positive, credibly non-zero push effect of the returning migrant stocks in the origin $\left(M_{j i}\right)$, as well as a negative, credibly non-zero pull effect of the migrant network in the destination $\left(M_{i j}\right)$ (Pedersen et al. 2008). The higher the common language level between the two countries $\left(L_{i j}\right)$, the more likely it was that migration movement between the two countries was observed. The unstructured time effects $\left(P_{t}\right)$, for which the 15 reference year was 2002 , were generally credibly higher than zero, which indicated, on average, an increase in the number of migration events over time (implied by the increase in the magnitude of the yearly coefficients, especially from 2006 onward). Finally, we found that the precision of the variance of the random effects $u_{i j}$ was quite large, which indicated a high

20 degree heterogeneity among the bilateral flows. Some bilateral flows were shown to be particularly strong, such as those between Germany and Poland, Italy and Romania, and France and the UK (Figure 3), which highlighted their importance at the European level.

\subsection{Case studies}

25 To better illustrate our results on the estimated migration flows, we examine six countries as case studies: i.e., Germany, Denmark, France, Poland, Sweden, and the United Kingdom. For each of these countries, we show the estimated total true latent flows for immigration (Figure 1), emigration (Figure 2), and net migration (Figure 3). Moreover, for comparison, we re30 port for the same flows the estimates from the IMEM project (Raymer et al. 2013), ranging from 2002 to 2008, as well as the total size of the respective flow from and to EU/EFTA countries (except for the reporting country) provided by Eurostat, which are known to suffer less of issues of incompleteness than the bilateral data. In the appendix, we show for nine selected 35 European countries the estimated total immigration (Figure 4), emigration (Figure 5), and net migration (Figure 6) flows; as well as the bilateral flows between all possible pairs (Figure 7).

France does not report any migration data by country of origin or destination in its administrative data. However, reliable immigration data for

40 France are available from the LFS (Martí \& Ródenas 2007). The Polish data reflect permanent migration only, and are, therefore, characterized by high levels of undercounting and low levels of accuracy. Sweden is a Nordic 
Table 1: Posterior characteristics of the parameters related to the countryspecific population coverage, $\operatorname{logit}^{-1}(\kappa)$ (except for the countries with excellent coverage), and the levels of undercounting, $v$.

\begin{tabular}{lccccc}
\hline \hline Parameter & $2.5 \%$ & $25 \%$ & $50 \%$ & $75 \%$ & $97.5 \%$ \\
\hline $\operatorname{logit}^{-1}\left(\kappa_{A T}\right)$ & 0.85 & 0.88 & 0.89 & 0.91 & 0.95 \\
$\operatorname{logit}^{-1}\left(\kappa_{B E}\right)$ & 0.96 & 0.99 & 0.99 & 1.00 & 1.00 \\
$\operatorname{logit}^{-1}\left(\kappa_{B G}\right)$ & 0.18 & 0.20 & 0.21 & 0.22 & 0.25 \\
$\operatorname{logit}^{-1}\left(\kappa_{C H}\right)$ & 0.95 & 0.98 & 0.99 & 1.00 & 1.00 \\
$\operatorname{logit}^{-1}\left(\kappa_{C Y}\right)$ & 0.01 & 0.41 & 0.83 & 0.97 & 1.00 \\
$\operatorname{logit}^{-1}\left(\kappa_{C Z}\right)$ & 0.35 & 0.38 & 0.40 & 0.43 & 0.47 \\
$\operatorname{logit}^{-1}\left(\kappa_{D E}\right)$ & 0.99 & 1.00 & 1.00 & 1.00 & 1.00 \\
$\operatorname{logit}^{-1}\left(\kappa_{E E}\right)$ & 0.39 & 0.43 & 0.45 & 0.47 & 0.51 \\
$\operatorname{logit}^{-1}\left(\kappa_{E S}\right)$ & 0.98 & 0.99 & 1.00 & 1.00 & 1.00 \\
$\operatorname{logit}^{-1}\left(\kappa_{F R}\right)$ & 0.01 & 0.37 & 0.82 & 0.97 & 1.00 \\
$\operatorname{logit}^{-1}\left(\kappa_{G R}\right)$ & 0.01 & 0.39 & 0.83 & 0.97 & 1.00 \\
$\operatorname{logit}^{-1}\left(\kappa_{H R}\right)$ & 0.28 & 0.30 & 0.32 & 0.33 & 0.37 \\
$\operatorname{logit}^{-1}\left(\kappa_{H U}\right)$ & 0.01 & 0.37 & 0.80 & 0.97 & 1.00 \\
$\operatorname{logit}^{-1}\left(\kappa_{I E}\right)$ & 0.95 & 0.98 & 0.99 & 1.00 & 1.00 \\
$\operatorname{logit}^{-1}\left(\kappa_{I T}\right)$ & 0.47 & 0.51 & 0.53 & 0.55 & 0.60 \\
$\operatorname{logit}^{-1}\left(\kappa_{L T}\right)$ & 0.92 & 0.98 & 0.99 & 1.00 & 1.00 \\
$\operatorname{logit}^{-1}\left(\kappa_{L U}\right)$ & 0.17 & 0.19 & 0.20 & 0.21 & 0.23 \\
$\operatorname{logit}^{-1}\left(\kappa_{L V}\right)$ & 0.01 & 0.38 & 0.80 & 0.97 & 1.00 \\
$\operatorname{logit}^{-1}\left(\kappa_{M T}\right)$ & 0.01 & 0.39 & 0.82 & 0.97 & 1.00 \\
$\operatorname{logit}^{-1}\left(\kappa_{P L}\right)$ & 0.10 & 0.11 & 0.12 & 0.12 & 0.14 \\
$\operatorname{logit}^{-1}\left(\kappa_{P T}\right)$ & 0.01 & 0.37 & 0.81 & 0.97 & 1.00 \\
$\operatorname{logit}^{-1}\left(\kappa_{R O}\right)$ & 0.01 & 0.39 & 0.82 & 0.97 & 1.00 \\
$\operatorname{logit}^{-1}\left(\kappa_{S I}\right)$ & 0.84 & 0.93 & 0.97 & 0.99 & 1.00 \\
$\operatorname{logit}^{-1}\left(\kappa_{S K}\right)$ & 0.13 & 0.14 & 0.15 & 0.15 & 0.17 \\
$\operatorname{logit}^{-1}\left(\kappa_{U K}\right)$ & 0.30 & 0.33 & 0.34 & 0.36 & 0.39 \\
$v_{1}$ & 0.78 & 0.84 & 0.86 & 0.89 & 0.94 \\
$v_{2}$ & 0.50 & 0.54 & 0.57 & 0.59 & 0.64 \\
$v_{3}$ & 0.74 & 0.80 & 0.82 & 0.84 & 0.89 \\
$v_{4}$ & 0.24 & 0.36 & 0.38 & 0.39 & 0.42 \\
$v_{5}$ & 0.31 & 0.33 & 0.35 & 0.36 & 0.38 \\
$v_{6}$ & & & & & \\
\hline \hline
\end{tabular}


Table 2: Posterior characteristics of the coefficients of the variables included in the migration model, $\gamma$, the precision of the random effect $u$; the level of accuracy in the immigration data, $\tau^{I R}$, and in the emigration data, $\tau^{E R}$.

\begin{tabular}{lccccc}
\hline \hline Parameter & $2.5 \%$ & $25 \%$ & $50 \%$ & $75 \%$ & $97.5 \%$ \\
\hline Intercept & -0.13 & -0.02 & 0.02 & 0.05 & 0.13 \\
$\log N_{i t}$ & -0.27 & -0.16 & 0.07 & 0.28 & 0.66 \\
$\log N_{j t}$ & -0.24 & -0.12 & 0.09 & 0.33 & 0.68 \\
$\log D_{i j}$ & -0.18 & -0.03 & 0.19 & 0.55 & 0.96 \\
$\log \left(G_{j t} / G_{i t}\right)$ & 0.71 & 0.75 & 0.77 & 0.79 & 0.84 \\
$A_{i j t}$ & -0.09 & -0.03 & 0.01 & 0.06 & 0.20 \\
$A_{i t}$ & 0.32 & 0.45 & 0.50 & 0.55 & 0.65 \\
$A_{j t}$ & 0.12 & 0.24 & 0.29 & 0.33 & 0.44 \\
$\log I_{i j t}$ & -0.43 & -0.15 & 0.09 & 0.16 & 0.33 \\
$\log E_{i j t}$ & -0.33 & -0.15 & -0.09 & 0.18 & 0.45 \\
$\log M_{i j}$ & 0.43 & 0.57 & 0.64 & 0.81 & 0.96 \\
$\log M_{j i}$ & 0.47 & 0.62 & 0.71 & 0.86 & 0.98 \\
$\log L_{i j}$ & 1.22 & 1.82 & 2.34 & 2.86 & 3.77 \\
$F_{i j t}$ & 0.30 & 0.34 & 0.35 & 0.37 & 0.39 \\
$P_{2002}$ & -0.04 & -0.005 & 0.01 & 0.03 & 0.06 \\
$P_{2003}$ & -0.16 & -0.12 & -0.10 & -0.08 & -0.05 \\
$P_{2004}$ & -0.12 & -0.08 & -0.06 & -0.05 & -0.02 \\
$P_{2005}$ & -0.05 & -0.01 & 0.01 & 0.02 & 0.05 \\
$P_{2006}$ & 0.0004 & 0.04 & 0.06 & 0.08 & 0.11 \\
$P_{2007}$ & 0.01 & 0.05 & 0.07 & 0.09 & 0.12 \\
$P_{2008}$ & -0.01 & 0.04 & 0.06 & 0.07 & 0.11 \\
$P_{2009}$ & 0.04 & 0.08 & 0.10 & 0.12 & 0.16 \\
$P_{2010}$ & 0.07 & 0.11 & 0.13 & 0.15 & 0.19 \\
$P_{2011}$ & 0.15 & 0.19 & 0.21 & 0.22 & 0.26 \\
$P_{2012}$ & 0.17 & 0.22 & 0.24 & 0.26 & 0.29 \\
$P_{2013}$ & 0.24 & 0.28 & 0.30 & 0.32 & 0.36 \\
$P_{2014}$ & 0.27 & 0.32 & 0.34 & 0.36 & 0.40 \\
$\tau_{u}$ & 5.75 & 6.51 & 6.95 & 7.41 & 8.41 \\
$\tau_{1}^{I R}$ & 19.16 & 24.35 & 27.55 & 31.23 & 40.34 \\
$\tau_{1}^{E R}$ & 19.07 & 23.57 & 26.44 & 29.78 & 37.67 \\
$\tau_{2}^{I R}$ & 12.40 & 13.40 & 14.01 & 14.66 & 16.08 \\
$\tau_{2}^{E R}$ & 8.01 & 8.52 & 8.82 & 9.15 & 9.81 \\
$\tau_{3}^{I R}$ & 1.00 & 1.05 & 1.08 & 1.10 & 1.15 \\
$\tau_{3}^{E R}$ & 0.86 & 0.90 & 0.92 & 0.94 & 0.98 \\
$\omega_{1}^{I S}$ & 0.80 & 0.85 & 0.88 & 0.90 & 0.97 \\
$\omega_{2}^{I S}$ & 0.53 & 0.55 & 0.56 & 0.57 & 0.60 \\
\hline \hline & & & & & \\
\hline \hline
\end{tabular}


country with a reliable data collection system, low levels of undercounting, excellent coverage, and high levels of accuracy. Finally, the UK derives its data not from population registers, but from the International Passenger Survey, a continuously running survey administered at the country's borders 5 (air, sea, and tunnel ports) that classifies respondents as long-term migrants if they intend to stay in the country for one year or more.

For all four countries, we observed increasing flows of both immigration and emigration following the EU enlargement in 2004 and the introduction of freedom of movement for workers in the EU (Figure 1 and Figure 2). However, among these selected countries, only Poland was found to have an increasingly negative net migration pattern, while all of the other countries reported highly positive net migration trends (Figure 3 ).

In France, immigration increased gradually over time, starting in 2004. Emigration also increased after two turning points: one in 2004, which marked the EU enlargement, and another in 2007, which corresponded to the start of the free movement in the labor market for workers from a number of Eastern European countries. Net migration was positive over the whole period, increasing from 10,000 to 30,000 people. The main immigrant groups in France were from the UK, Italy, and Spain (Figure 7). The inflows from all of these countries increased over time. The degree of attractiveness of the UK and France appeared to be similar, as the migration flows between the two countries were fairly comparable.

In Poland, immigration showed an increasing trend, with an abrupt change occurring in 2007, when inflows from Germany and the UK in partic25 ular grew rapidly. Given that these two countries were also the leading destinations for migrants from Poland, it is likely that these inflows to Poland mainly consisted of return migrants (Figure 7). Polish emigration increased abruptly at two points in time: one in 2004, after the country entered the EU; and one in 2011, when Polish workers were first allowed to freely enter the German labor market. Looking at the net migration patterns, we notice that over time, Poland generally experienced a pattern of increasingly negative net migration, with turning points occurring at the peaks in immigration.

Sweden also experienced a surge in immigration from 2004 onward, with immigration reaching a peak between 2007 and 2008, and gradually increasing thereafter. The net migration trend in Sweden was positive and increasing over time, with turning points occurring in 2004 and in 2009. As the information on migration patterns in Sweden mainly comes from highquality administrative data, the estimates follow the time series provided by 40 Eurostat quite nicely.

Finally, the trends observed in the UK were similar to those reported in Sweden, except that the numbers were considerably larger. The net migration trend for this period in the UK was consistently positive, with the increasing pattern beginning in 2004, reaching a peak in 2007, and hitting 
a low point in 2011. The main countries of immigration to the UK were Poland, France, Spain, and Italy (Figure 7).

When considering the comparison with the IMEM estimates, at least for the period between 2002 and 2008, we found that a high degree of consis-

5 tency, especially for Sweden and the UK. The main differences were found for France (immigration, emigration, and net migration, for Poland (immigration), and for Sweden (net migration). On the other hand, we contrasting our estimates with the data reported by Eurostat on the total immigration and the emigration from and to EU/EFTA countries, the differences were more striking, as the marginal Eurostat data suffer, as well as the bilateral data, problems of inconsistency and, to a lesser extent, incompleteness.

Among the other five selected countries shown in the Appendix, we noticed relevant negative net migration trends for Poland, Romania, and, to a lesser extent, for Spain and Italy. Conversely, the highest positive net 15 migration patterns were found for Germany and the UK (Figure 6). The larger differences with the IMEM estimates, evident when looking at the net migration numbers, were found for Spain, Italy, and the Netherlands.

\section{Conclusions}

We developed a hierarchical Bayesian model to estimate the true latent international migration flows from 2002 to 2015 among 31 European countries, with the data informing the model coming from administrative data sources and from the EU Labour Force Surveys. A measurement error model was used to harmonize the data from the different data sources and to account for the bias in the measurements induced by the data inconsistencies. We then used a migration model based on migration theory and on empirical evidence to predict the true latent migration flows, including for those countries lacking data.

The novelty of our work can be appreciated under three dimensions. First, we showed that for the measurement of the international migration in Europe, different data sources that provide information on international migration flows among European countries are available: namely, population registers and other administrative sources, household surveys such as the Labor Force Survey, and population censuses. Focusing on the first two sources, we showed that they usually suffer from issues of incompleteness and inconsistencies. The inconsistencies are related to the time dimension, which can vary depending on which definition of long-term migration is used; the undercounting bias; the degree of population coverage; the accuracy of the data collection system; and, for the LFS, the sampling design. Despite these issues, we showed that combining the information provided by these data sources can be an effective approach, provided the incompleteness of and the inconsistencies in these data are properly taken into consideration 
FR

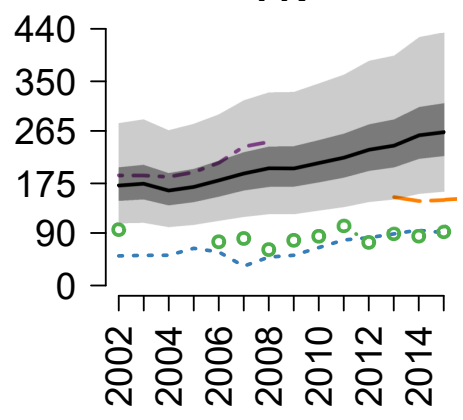

SE

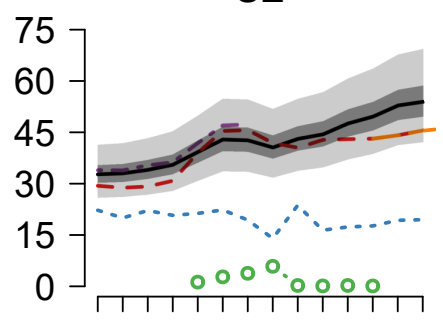

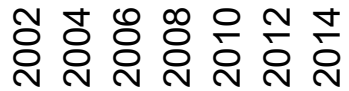

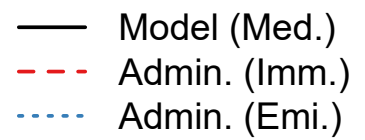

PL

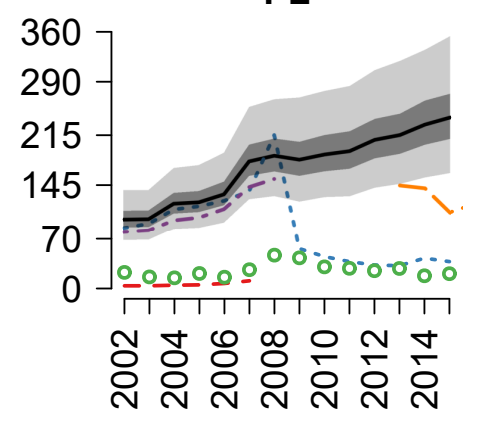

UK

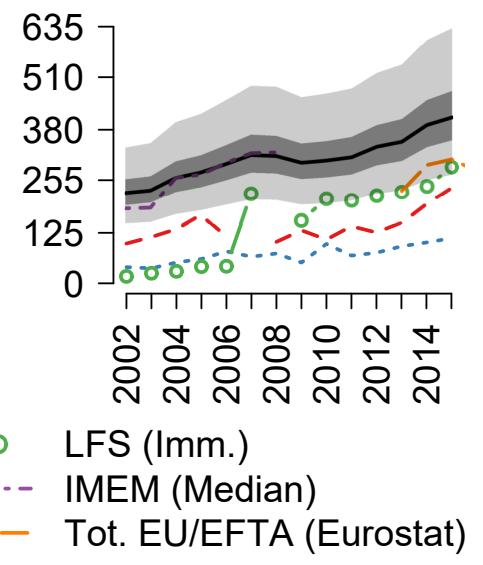

Figure 1: Immigration: Estimated total flow (in thousands) with 50\% CI (dark gray) and 95\% CI (light gray) for France (FR), Poland (PL), Sweden (SE), and the United Kingdom (UK). We also reported the summed flows from administrative immigration data (dashed red line), from administrative emigration data (dotted blue lines), from LFS immigration data (green circles). For comparison, we reported the median of the estimates from the IMEM project (Raymer et al. 2013) (2002-2008, dash-and-dot purple line) and the total immigration from EU/EFTA as reported by Eurostat (large dashed orange lines). 
FR

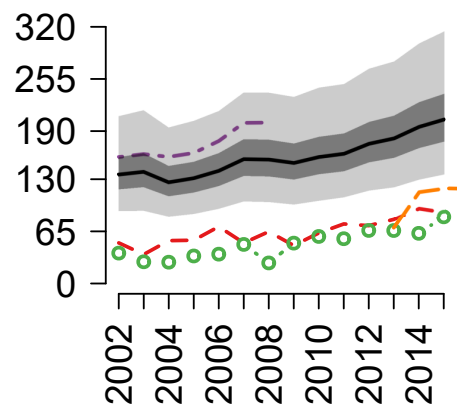

SE

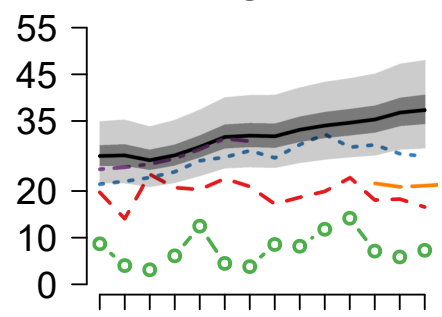

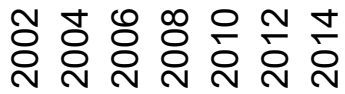

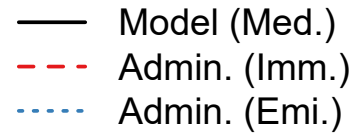

PL

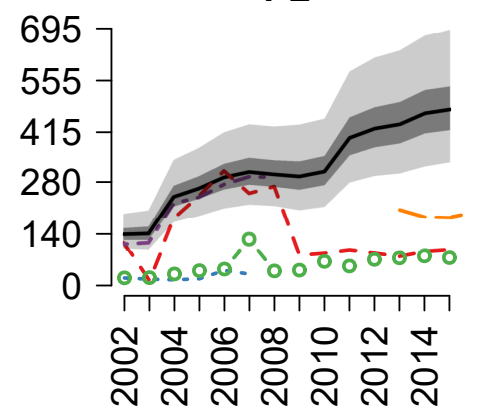

UK

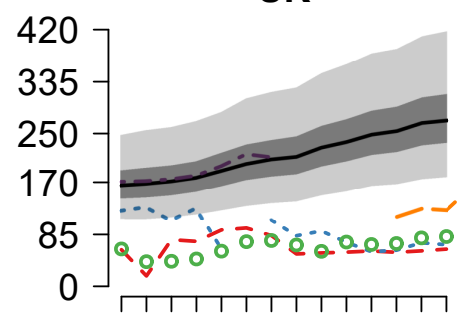

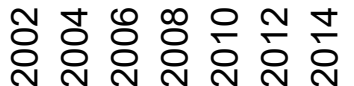

$\circ \quad$ LFS (Imm.)

IMEM (Median)

Tot. EU/EFTA (Eurostat)

Figure 2: Emigration: Estimated total flow (in thousands) with 50\% CI (dark gray) and 95\% CI (light gray) for France (FR), Poland (PL), Sweden (SE), and the United Kingdom (UK). We also reported the summed flows from administrative immigration data (dashed red line), from administrative emigration data (dotted blue lines), from LFS immigration data (green circles). For comparison, we reported the median of the estimates from the IMEM project (Raymer et al. 2013) (2002-2008, dash-and-dot purple line) and the total immigration from EU/EFTA as reported by Eurostat (large dashed orange lines). 
FR

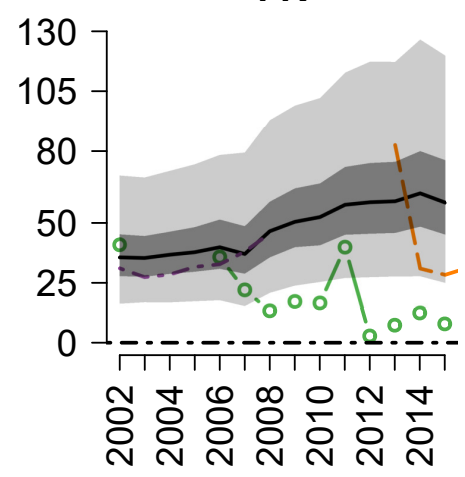

SE

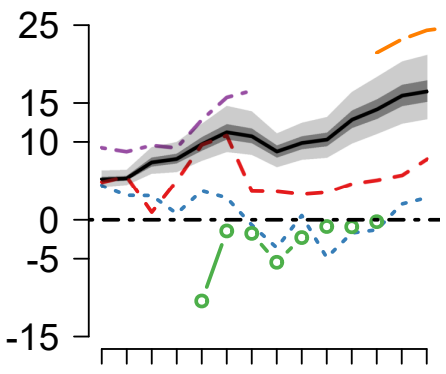

del (Med.)

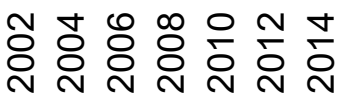

pReg (Dest.)

.... PopReg (Orig.)

- LFS (Dest.)
PL

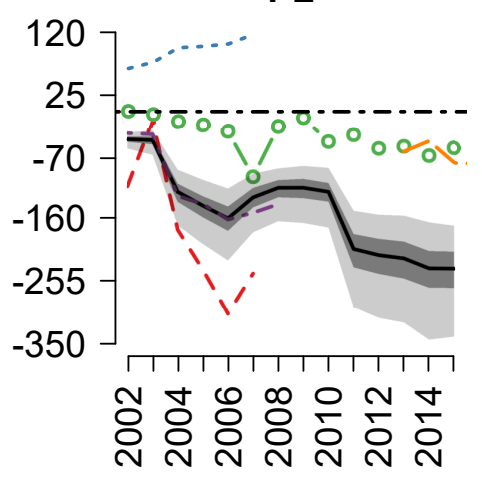

UK

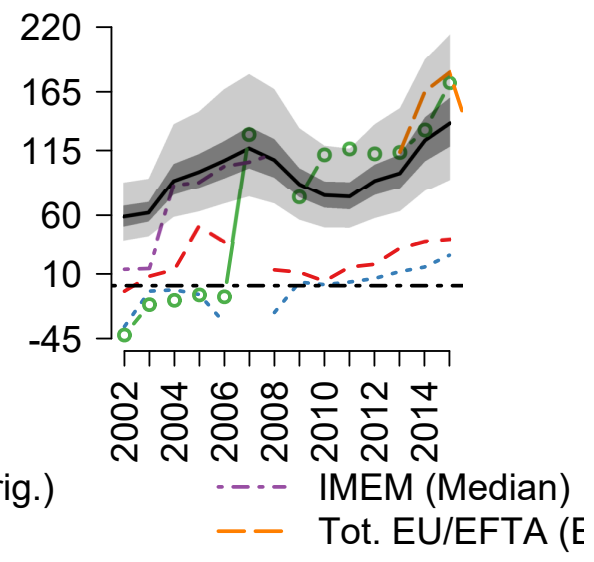

Figure 3: Net migration: Estimated total flow (in thousands) with $50 \%$ CI (dark gray) and 95\% CI (light gray) for France (FR), Poland (PL), Sweden (SE), and the United Kingdom (UK). We also reported the summed flows from administrative immigration data (dashed red line), from administrative emigration data (dotted blue lines), from LFS immigration data (green circles). For comparison, we reported the median of the estimates from the IMEM project (Raymer et al. 2013) (2002-2008, dash-and-dot purple line) and the total immigration from EU/EFTA as reported by Eurostat (large dashed orange lines). The horizontal black dashed-dotted line indicates a level of net migration equal to zero. 
in the modeling framework.

Second, in terms of data processing, we showed how multiple data sources can be integrated into a single hierarchical Bayesian statistical model. This model is not only based on the multiple data sources on migration mentioned 5 above, it also accommodates auxiliary data that can be used to assess the relative attractiveness of pairs of countries, as well as the quality of the data, converted into probability statements in the form of prior distributions for the parameters. An important limitation of our work regards especially the definition of the prior distributions and the grouping of countries functional to the use of such priors. Some of the priors distributions, especially those relative to the measurement error models for the administrative data, are based on assumptions first formulated in the paper by Raymer et al. (2013); other are based on our beliefs, mainly because of the observation that use of elicited expert opinion proved to be quite non informative (Wiśniowski et al. 2013, Willekens 2019). Moreover, the countries' grouping in terms of undercounting, population coverage, and data collection accuracy are taken as well from Raymer et al. (2013) and assumed to be time-invariant. Such an assumption might be quite strong, as the quality of statistics is not constant in time and it is likely that estimates for an early period cannot be really used for the most recent data. Further information on how the various national statistical institutes changed their data collection system over time could lead to better countries' groupings and prior distribution definitions and, in turn, to more realistic and accurate estimates. Our model builds upon previously published Bayesian models that were separately developed (Raymer et al. 2013, Wiśniowski 2017)). The key rationale for combining the different data sources is to estimate the latent true relocation rate (Nowok \& Willekens 2011), and then to use it to predict the true latent migration flows, conditional on the recommended criterion of defining a long-term migration event as having a minimum duration of 12 months. Another innovation of our work is that we extended the period under study by using all the available administrative and LFS data from 2002 to 2015 (while previous work provided estimates up to 2008 only).

Third, the results of our modeling framework confirmed that our estimates reflected the impact of the EU enlargement (2004) and the extension of the freedom of movement for workers within the EU (2007) on intraEuropean migration patterns. As expected, we found that the level of uncertainty around the estimates of the true latent migration flows largely depended on the amount of available information. Our results also indicated 40 that the variability was higher for countries that often reported incomplete time series of data (e.g., France, Poland, Romania, and Germany) or that used less reliable data sources, such as sampling surveys (UK). On the other hand, we found that the variability was much lower for countries like Sweden and the Netherlands because of the better quality of their migration data. 
Thus, the true latent migration flow for these countries was shown to be closer to the reported data, especially to the data from the administrative sources.

We believe that our Bayesian statistical framework is flexible enough 5 to be extended through the inclusion of new data sources on migration flows, new variables for the migration model, or new information that allow to construct better prior distributions for the parameters of interest. We could, for example, augment the data sources we currently use with data from social media - e.g., geo-referenced Twitter data (Zagheni et al. 2014) 10 or Facebook network data (Spyratos et al. 2019) - or with data from the IP addresses of email service providers (Zagheni \& Weber 2012).

Alternatively, we could add new variables to the migration model. It would, for example, be interesting to explore the possibility of including online search data in the model as a proxy for migration intentions (Böhme et al. 2019)). Data on income inequality, living conditions, unemployment, or social expenditures could be also included in the model as possible push effects for migration intentions (Mayda 2010). Finally, the model could be further extended by stratifying the migration data by gender and age to help us gain a better understanding of the drivers of population change and the heterogeneity within migrant groups (Wiśniowski et al. 2016).

A further enhancement could come from the collection of more precise information on the level of incompleteness and inconsistency in the migration data, as a way to improve our assessment of data quality. For this purpose, we are planning to involve experts from different national statistical offices in Europe to understand how statistical offices adapted their collection system to the EU Regulation No. 862/2007 before delivering migration data to Eurostat. In particular, we aim to investigate whether they made any change in the data sources used or in the definition of migration events, and whether they made any assessment of the level of bias in the reporting of in-flows and out-flows. We believe that this inquiry would enable us to build more precise prior distributions for the model parameters, especially for those in the measurement error model.

\section{Acknowledgements}

We thank Frans Willekens and Dmitri Jdanov for their valued feedback on 35 an earlier draft of the paper. We also thank Karolin Kubisch for assisting us in the process of obtaining the access to the LFS data.

\section{References}

Abel, G. J. (2010), 'Estimation of international migration flow tables in Europe: International Migration Flow Tables', Journal of the Royal Sta- 
tistical Society: Series A (Statistics in Society) 173(4), 797-825.

Abel, G. J. (2013), 'Estimating global migration flow tables using place of birth data', Demographic Research 28(18), 505-546.

Abel, G. J. \& Cohen, J. E. (2019), 'Bilateral international migration flow estimates for 200 countries', Scientific Data 6(1), 1-13.

Abel, G. J. \& Sander, N. (2014), 'Quantifying Global International Migration Flows', Science 343(6178), 1520-1522.

Azose, J. J. \& Raftery, A. E. (2019), 'Estimation of emigration, return migration, and transit migration between all pairs of countries', Proceedings of the National Academy of Sciences 116(1), 116-122.

Bijak, J. \& Bryant, J. (2016), 'Bayesian demography 250 years after Bayes', Population Studies 70(1), 1-19.

Böhme, M. H., Gröger, A. \& Stöhr, T. (2019), 'Searching for a better life: Predicting international migration with online search keywords', Journal of Development Economics p. 102347.

Cohen, J. E., Roig, M., Reuman, D. C. \& GoGwilt, C. (2008), 'International migration beyond gravity: A statistical model for use in population projections', Proceedings of the National Academy of Sciences 105(40), 1526915274 .

20 Kupiszewska, D. \& Nowok, B. (2008), Comparability of Statistics on International Migration Flows in the European Union, in 'International Migration in Europe', John Wiley \& Sons, Ltd, pp. 41-71.

Maasing, E., Tiit, E.-M. \& Vähi, M. (2017), 'Residency index - a tool for measuring the population size', Acta et Commentationes Universitatis Tartuensis de Mathematica 21(1), 129-139.

Martí, M. \& Ródenas, C. (2007), 'Migration Estimation Based on the Labour Force Survey: An EU-15 Perspective', International Migration Review 41(1), 101-126.

Matthews, R. A. J. (2019), 'Moving Towards the Post $\mathrm{p}<0.05$ Era via the Analysis of Credibility', The American Statistician 73(sup1), 202-212.

Mayda, A. M. (2010), 'International migration: a panel data analysis of the determinants of bilateral flows', Journal of Population Economics 23(4), 1249-1274.

Mayer, T. \& Zignago, S. (2011), 'Notes on CEPII's Distances Measures: The GeoDist Database', SSRN Electronic Journal. 
Melitz, J. \& Toubal, F. (2014), 'Native language, spoken language, translation and trade', Journal of International Economics 93(2), 351-363.

Nowok, B. (2007), Evolution of International Migration Statistics in Selected Central European Countries, in J. Raymer \& F. Willekens, eds, 'International Migration in Europe', John Wiley \& Sons, Ltd, Chichester, UK, pp. $73-87$.

Nowok, B. (2010), Harmonization by simulation: a contribution to comparable international migration statistics in Europe, $\mathrm{PhD}$ thesis, University of Groningen, Groningen, Netherlands. OCLC: 955134815.

Nowok, B. \& Willekens, F. (2011), 'A probabilistic framework for harmonisation of migration statistics: Harmonisation of Migration Statistics', Population, Space and Place 17(5), 521-533.

Özden, c., Parsons, C. R., Schiff, M. \& Walmsley, T. L. (2011), 'Where on Earth is Everybody? The Evolution of Global Bilateral Migration 1960-2000', The World Bank Economic Review 25(1), 12-56.

Pedersen, P. J., Pytlikova, M. \& Smith, N. (2008), 'Selection and network effects-Migration flows into OECD countries 1990-2000', European Economic Review 52(7), 1160-1186.

Plummer, M. (2003), JAGS: A program for analysis of Bayesian graphical models using Gibbs sampling, in 'Proceedings of the 3rd international workshop on distributed statistical computing', Vol. 1243, Vienna, Austria, p. 10.

Plummer, M., Best, N., Cowles, K. \& Vines, K. (2006), 'Coda: Convergence diagnosis and output analysis for mcmc', R News 6(1), 7-11.

Raymer, J. \& Wiilekens, F. (2008), International Migration in Europe: Data, Models and Estimates, John Wiley \& Sons.

Raymer, J., Wiśniowski, A., Forster, J. J., Smith, P. W. F. \& Bijak, J. (2013), 'Integrated Modeling of European Migration', Journal of the American Statistical Association 108(503), 801-819.

Schmertmann, C. P. (1999), 'Estimating Multistate Transition Hazards from Last-Move Data', Journal of the American Statistical Association 94(445), 53-63.

Spyratos, S., Vespe, M., Natale, F., Weber, I., Zagheni, E. \& Rango, M. (2019), 'Quantifying international human mobility patterns using Facebook Network data', PLOS ONE 14(10), e0224134. 
United Nations Statistics Division (1998), Recommendations on statistics of international migration. Revision 1, United Nations Publications, New York.

Willekens, F. (1994), 'Monitoring international migration flows in Europe', European Journal of Population/Revue européenne de Démographie 10(1), 1-42.

Willekens, F. (2019), 'Evidence-Based Monitoring of International Migration Flows in Europe', Journal of Official Statistics 35(1), 231-277.

Willekens, F., Massey, D., Raymer, J. \& Beauchemin, C. (2016), 'International migration under the microscope', Science 352(6288), 897-899.

Wiśniowski, A. (2017), 'Combining Labour Force Survey data to estimate migration flows: the case of migration from Poland to the UK', Journal of the Royal Statistical Society: Series A (Statistics in Society) 180(1), 185202.

Wiśniowski, A., Forster, J. J., Smith, P. W. F., Bijak, J. \& Raymer, J. (2016), 'Integrated modelling of age and sex patterns of European migration', Journal of the Royal Statistical Society: Series A (Statistics in Society) 179(4), 1007-1024.

Wiśniowski, A., Bijak, J., Christiansen, S., Forster, J. J., Keilman, N., Raymer, J. \& Smith, P. W. (2013), 'Utilising Expert Opinion to Improve the Measurement of International Migration in Europe', Journal of Official Statistics 29(4), 583-607.

Zagheni, E., Garimella, V. R. K., Weber, I. \& State, B. (2014), Inferring international and internal migration patterns from Twitter data, in 'Proceedings of the 23rd International Conference on World Wide Web WWW '14 Companion', ACM Press, Seoul, Korea, pp. 439-444.

Zagheni, E. \& Weber, I. (2012), You are where you e-mail: using e-mail data to estimate international migration rates, in 'Proceedings of the 3rd Annual ACM Web Science Conference on - WebSci '12', ACM Press, Evanston, Illinois, pp. 348-351. 

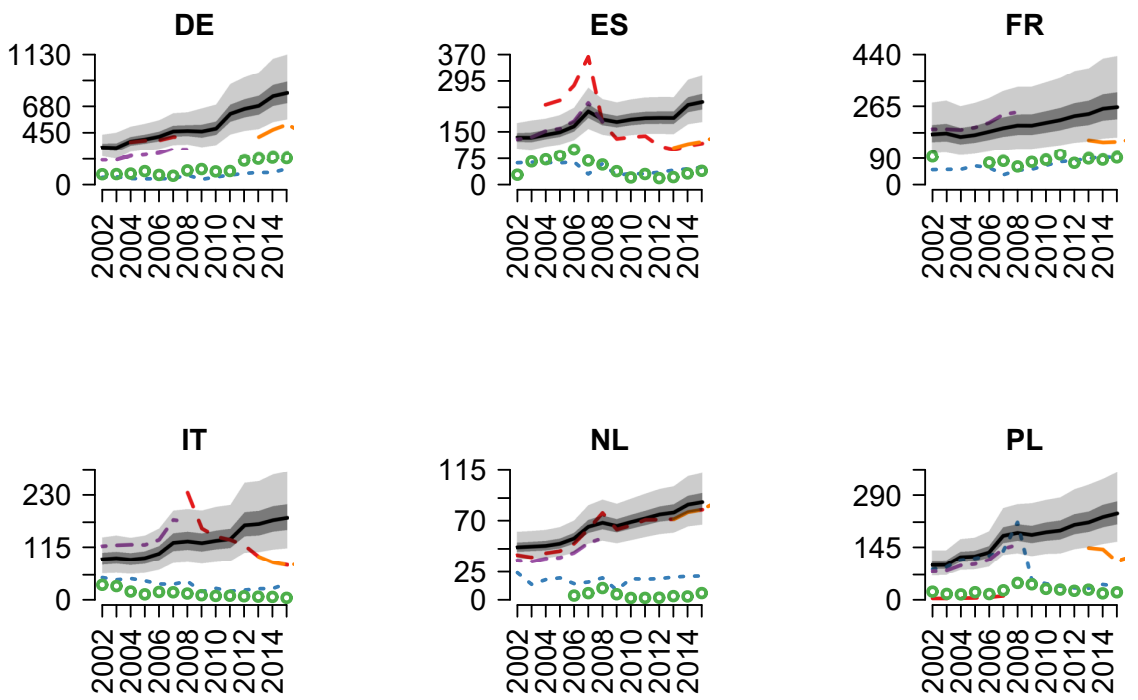

RO

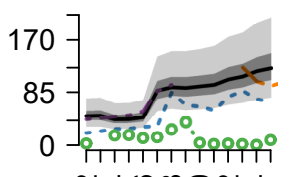

껑잉은ㄷㄴ
SE

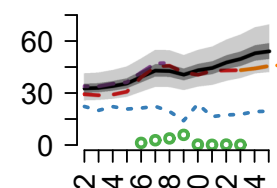

UK

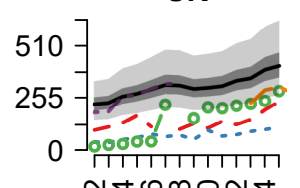

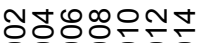

Figure 4: Immigration: Estimated total flow (in thousands) with 50\% CI (dark gray) and 95\% CI (light gray) for nine European countries. We also reported the summed flows from administrative immigration data (dashed red line), from administrative emigration data (dotted blue lines), from LFS immigration data (green circles). For comparison, we reported the median of the estimates from the IMEM project (Raymer et al. 2013) (2002-2008, dash-and-dot purple line) and the total immigration from EU/EFTA as reported by Eurostat (large dashed orange lines). 

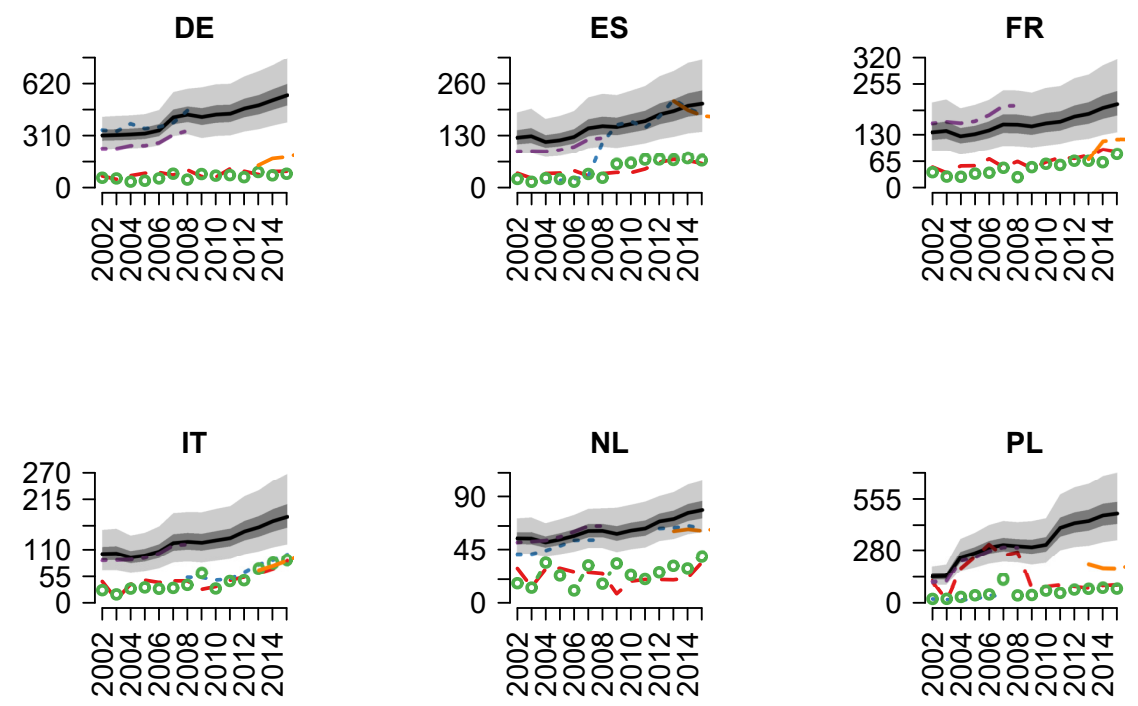

NL

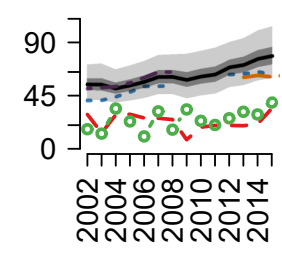

PL

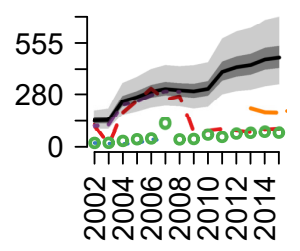

RO

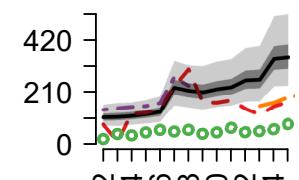

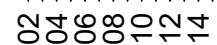

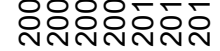

- Model (Med.)

- Admin. (Imm.)

.... Admin. (Emi.)
SE

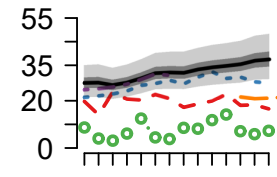

ํํㅇํํ융ํํํํํำ
UK

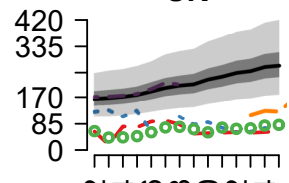

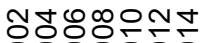

Figure 5: Emigration: Estimated total flow (in thousands) with 50\% CI (dark gray) and 95\% CI (light gray) for nine European countries. We also reported the summed flows from administrative immigration data (dashed red line), from administrative emigration data (dotted blue lines), from LFS immigration data (green circles). For comparison, we reported the median of the estimates from the IMEM project (Raymer et al. 2013) (2002-2008, dash-and-dot purple line) and the total immigration from EU/EFTA as reported by Eurostat (large dashed orange lines). 

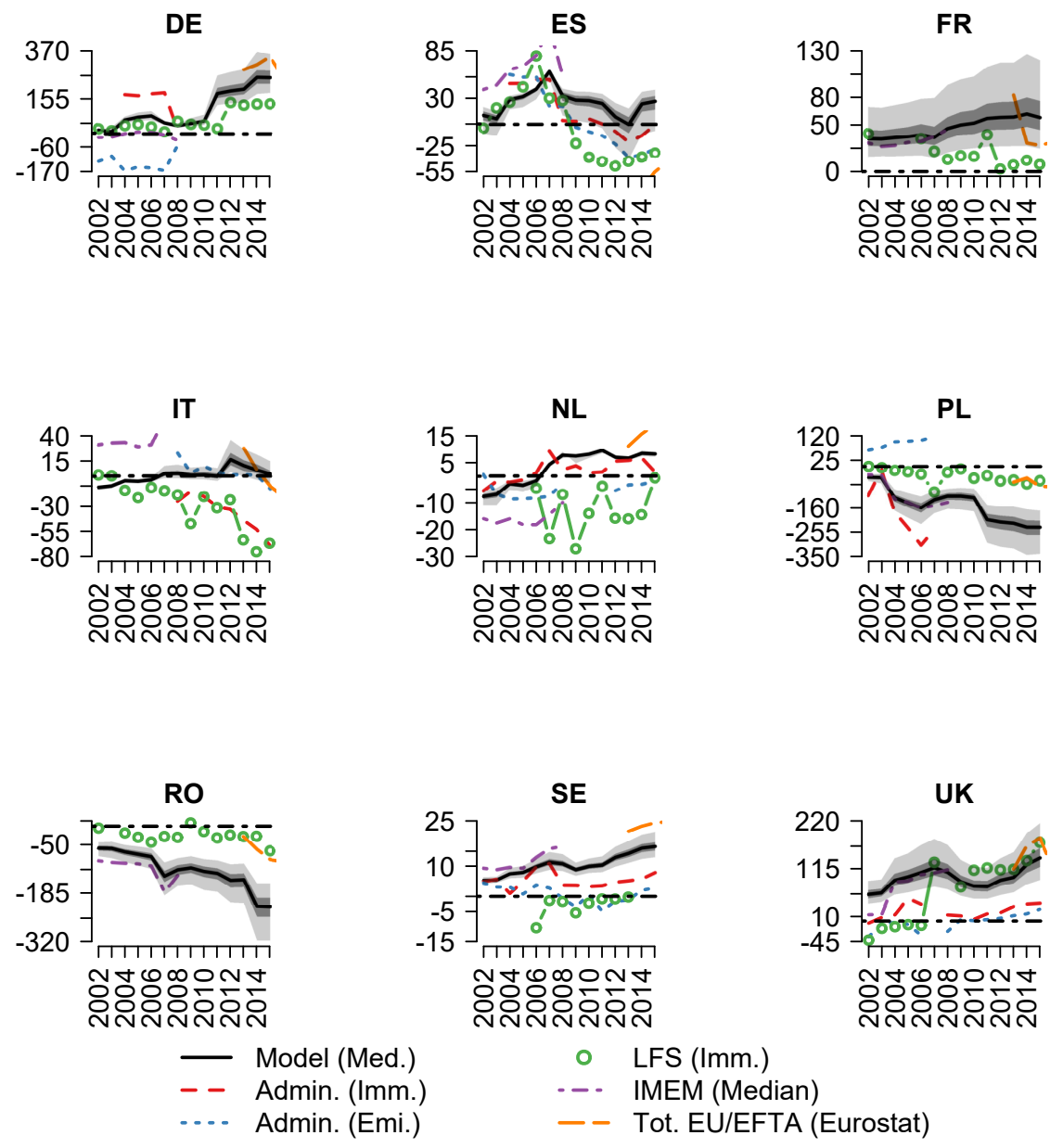

Figure 6: Net migration: Estimated total flow (in thousands) with 50\% CI (dark gray) and 95\% CI (light gray) for nine European countries. We also reported the summed flows from administrative immigration data (dashed red line), from administrative emigration data (dotted blue lines), from LFS immigration data (green circles). For comparison, we reported the median of the estimates from the IMEM project (Raymer et al. 2013) (2002-2008, dashand-dot purple line) and the total immigration from EU/EFTA as reported by Eurostat (large dashed orange lines). The horizontal black dashed-dotted line indicates a level of net migration equal to zero. 


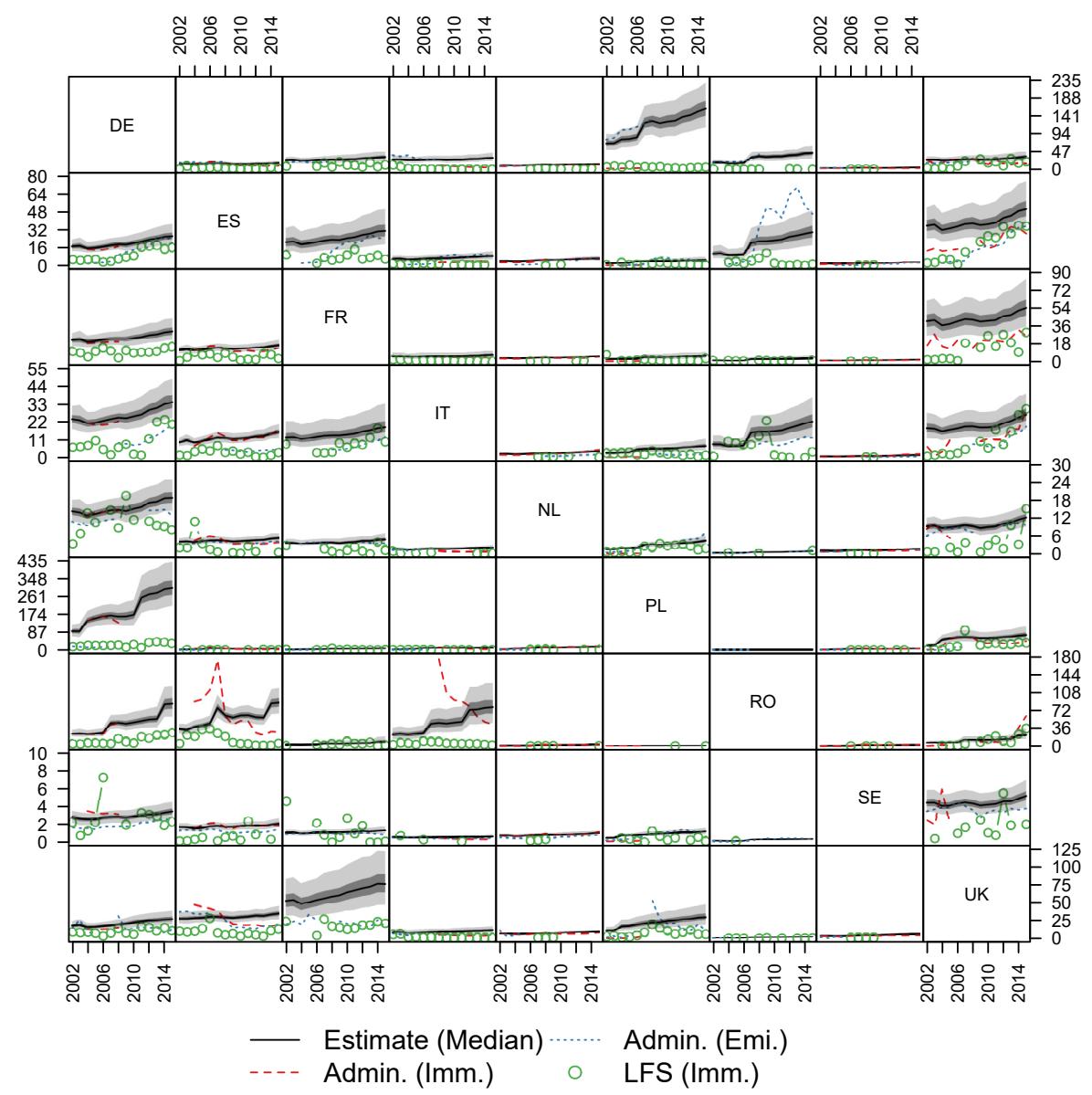

Figure 7: Estimated bilateral flows (in thousands) with 9\% CI (dark gray) and $95 \%$ CI (light gray) for nine European countries. In addition, immigration flows (dashed line) and emigration flows (dotted line) from the administrative data sources, and immigration flows from the LFS data (circles). 